\title{
The Epithelial Barrier Hypothesis: a 20-year journey
}

\author{
Yağız Pat ${ }^{1}$ and ISMAIL OGULUR ${ }^{1}$ \\ ${ }^{1}$ University of Zurich
}

May 5, 2021

News \& Views: Groundbreaking Discoveries in Immunology
The Epithelial Barrier Hypothesis: a 20-year journey

Yagiz Pat ${ }^{1,2^{*}}$, Ismail Ogulur ${ }^{1,3^{*}}$

1 Swiss Institute of Allergy and Asthma Research (SIAF), University of Zurich, Davos, Switzerland

2 Department of Medical Microbiology, Faculty of Medicine, Aydın Adnan Menderes University, Aydın, Turkey

3 Division of Pediatric Allergy and Immunology, School of Medicine, Marmara University, Istanbul, Turkey

*Corresponding authors:

Yagiz Pat, Swiss Institute of Allergy and Asthma Research (SIAF), Herman Burchard Str. 9, 7265 Davos Wolfgang, Switzerland, e-mail: yagizpat@gmail.com

Ismail Ogulur, Swiss Institute of Allergy and Asthma Research (SIAF), Herman Burchard Str. 9, 7265 Davos Wolfgang, Switzerland, e-mail: ismail.ogulur@gmail.com

Keywords: Epithelial barrier hypothesis, allergic diseases, autoimmune diseases, metabolic diseases, leaky gut, microbial dysbiosis, tissue microinflammation, type 1 immunity, type 2 immunity

Conflict of Interest: Dr Pat and Dr Ogulur have nothing to disclose.

Acknowledgement: We would like to thank Dr Anna Globinska for assistance in generating the graphical figure.

\section{Main Text}

The global environment has been transformed rapidly in the last six decades as a direct result of a more westernized, industrialized, and modernized world. At the same time, humanity has faced a marked increase in allergic, autoimmune, metabolic and certain neuropsychiatric diseases, especially in industrialized countries. It is now evident that exposure to toxic substances together with lifestyle changes due to urbanization, increased early use of antibiotics, a westernized diet, and obesity play important roles in the development of these diseases. ${ }^{1}$ Despite the extensive progress in understanding the pathophysiology of allergic and autoimmune diseases, there is no comprehensive view that can fully explain the reasons behind the epidemic increase of these conditions worldwide. A comprehensive review article, introducing "The Epithelial Barrier Hypothesis" which aimed to elucidate this phenomenon, was recently published. ${ }^{1}$ This hypothesis postulates that environmental exposure to toxic substances introduced by modern lifestyle affects the epithelial barrier of the skin, upper and lower airways and gut mucosa. These substances include detergents, cleaning products (particularly enzymes and surfactants), microplastics, nanoparticles, increased ozone and particulate matter concentrations, cigarette smoke, and food additives (enzymes and emulsifiers). Exposure to these harmful agents leads to the development of leaky epithelial barriers, microbial dysbiosis, translocation of bacteria 
to inter- and sub-epithelial areas, and tissue microinflammation in and around the epithelial barriers. "The Epithelial Barrier Hypothesis" also proposes that the barrier damage caused by the environmental changes is not only responsible for the development of allergy and autoimmune conditions, but also a wide range of diseases in which immune responses to translocated bacteria have systemic effects. ${ }^{1}$ This list can be extended by further investigating diseases with microbial dysbiosis and subepithelial translocation of bacteria and immune response to commensals or opportunistic bacteria.

The Epithelial Barrier Hypothesis is rooted in studies from the beginning of the century that demonstrate immune-mediated epithelial barrier damage in chronic allergic inflammation. One of the first findings regarding the immune-mediated epithelial barrier damage was made in atopic dermatitis and allergic contact dermatitis patients. Skin infiltrating T cells were demonstrated to induce keratinocyte apoptosis leading to eczema development, a skin barrier defect. ${ }^{2}$ Since then, many studies have elaborated on the concept of type 2 immunity mediated barrier damage in various diseases such as asthma, chronic rhinosinusitis, and colitis. ${ }^{1-3}$ Type 2 immunity, which is the default defence against parasites and venoms, plays a crucial role in epithelial barrier regulation. It was proposed in 2006 that the thickening of the airway basement membrane forms a barrier below the leaky epithelium between the environment that may include a dysbiotic microbiome and toxic substances and inner tissues to prevent the entry of harmful components. ${ }^{3}$ Factors that "keep away" the environmental knox and that "wash away" the toxic subtances together with inflammation have been proposed. The opening of epithelial barriers, increased mucus production and mucosal shedding, representatives of a mechanism that attempts to reduce allergen exposure (wash-away effect). ${ }^{1,3}$ It is well known that type 2 cytokines such as IL-4 and IL-13 open the epithelial barriers. ${ }^{4}$ Conditions characterized by a systemic type 2 immune response, such as asthma, chronic rhinosinusitis, and atopic dermatitis, are well-defined by an impaired epithelial barrier. ${ }^{1,4}$ "The Epithelial Barrier Hypothesis" takes into account five facts; the steep increase in allergic and autoimmune diseases, the evidence of epithelial barrier disruption in these conditions, microbial dysbiosis and bacterial translocation, immune response to commensals and opportunistic pathogens, and changes in environmental exposure due to urbanization and industrialization. ${ }^{1}$ In the last century, humanity has faced new environmental challenges including poor air quality, climate change, and increased use of toxic substances such as detergents, nanoparticles, and microplastics. These biological and chemical insults disrupt the barrier integrity and cause the release of epithelial cytokines such as IL-25, IL-33, and thymic stromal lymphopoietin which leads to a type 2 immune response in affected organs in asthma, rhinitis, chronic rhinosinusitis, eosinophilic esophagitis, food allergy, and atopic dermatitis. ${ }^{5,6}$

The Epithelial Barrier Hypothesis presents an overarching idea that also embraces previous views from the Hygiene, Old Friends, and Biodiversity hypotheses. ${ }^{1}$ It includes all previous mechanisms and brings a promising rationale to explain the sudden increase of chronic noncommunicable inflammatory diseases observed in the last six decades. It offers new ideas for diagnosis, treatment, and prevention of diseases associated with epithelial barrier leakiness. Moreover, the hypothesis further introduces novel mechanisms for the pathophysiology of autoimmune diseases, chronic neuropsychiatric conditions, and metabolic diseases.

\section{References}

1. Akdis CA. Does the epithelial barrier hypothesis explain the increase in allergy, autoimmunity and other chronic conditions? Nat Rev Immunol. 2021.

2. Trautmann A, Akdis M, Kleemann D, et al. T cell-mediated Fas-induced keratinocyte apoptosis plays a key pathogenic role in eczematous dermatitis. J Clin Invest. 2000;106(1):25-35.

3. Akdis CA. Allergy and hypersensitivity: mechanisms of allergic disease. Curr Opin Immunol. 2006;18(6):718-726.

4. Soyka MB, Wawrzyniak P, Eiwegger T, et al. Defective epithelial barrier in chronic rhinosinusitis: the regulation of tight junctions by IFN-gamma and IL-4. J Allergy Clin Immunol. 2012;130(5):1087-1096 e1010.

5. Akdis CA, Arkwright PD, Bruggen MC, et al. Type 2 immunity in the skin and lungs. Allergy. 2020;75(7):1582-1605. 
6. Celebi Sozener Z, Cevhertas L, Nadeau K, Akdis M, Akdis CA. Environmental factors in epithelial barrier dysfunction. J Allergy Clin Immunol. 2020;145(6):1517-1528.

\section{Figure legend:}

The Epithelial Barrier Hypothesis proposes that environmental exposure to toxic substances affects the epithelial cells and leads to the development of leaky epithelial barriers, microbial dysbiosis, translocation of bacteria to inter- and sub-epithelial areas, and tissue microinflammation, and immune response to opportunistic pathogens and commensals. It also points out a possible link between barrier disruption and inflammation at distant organs, such as joints and central nervous system, due to migration of activated immune cells to target organs. 